\title{
Modal Propellant Gauging
}

\author{
Jackson Wehr, Nathaniel Lee, Megan Janiak \\ Affiliation (Physics, Carthage College) \\ Kenosha, WI, United States
}

\begin{abstract}
Since 2011, the Modal Propellant Gauging (MPG) team, consisting of multidisciplinary undergraduate researchers from Carthage College, has been developing and testing a fuel gauging system for use in microgravity environments. Using experimental modal analysis (EMA) techniques, the goal of the MPG project is to develop a flight ready technology that gauges fuel in microgravity environments by correlating the modal response of a 1-g equilibrium surface to the microgravity surface response at the same fluid fill level. The technology has been tested aboard parabolic flights via a manned parabolic flight payload. The payload consists of two propellant tanks and is designed to measure the modal response of each propellant tank to an injected white noise signal via piezoelectric sensors. Flight data shows that the MPG method can measure fuel with greater than or equal to $1 \%$ resolution at and below $50 \%$ fill levels. Under funding from the Wisconsin Space Grant Consortium during the summer of 2017, the MPG team made improvements to the parabolic flight payload in addition to designing a new payload for use aboard a Blue Origin New Shepard research flight scheduled for the first half of 2018.
\end{abstract}

\section{Introduction}

The Modal Propellant Gauging (MPG) project serves the purpose to develop a higher resolution fuel gauging method for microgravity environments. This paper acts both as a general overview of the project as well as a report on the work done during the past year, especially the recent summer months. The work on MPG has been done heavily by students with the direction, guidance and help of Dr. Kevin Crosby. There are two specific sub-projects within this overarching initiative. One is for testing the technology on parabolic flights, the other is to truly test the original hypothesis via a Blue Origin New Shepard Mission.

1.1. Program The MPG project has been around at Carthage College since 2011. There have been multiple versions of the payload rig, each with some new features to further advance the technology. Students flew on parabolic flights with Dr. Kevin Crosby through NASA until recent years. The last few flight campaigns have been through Zero-G Corporation. The current students on the project have only flown through Zero-G. There were two flight campaigns and there will be one more in November of 2017.

1.2. Purpose Fuel gauging in microgravity environments has always been difficult. It is very challenging to acquire a good resolution of fuel in microgravity environments largely due to the behavior of liquid. International requirements enforce satellites to be pushed into a graveyard orbit at the end of their lives. Due to poor resolution of fuel gauging, many satellites are often kicked out prematurely. The typical resolutions of common methods, such as book-keeping and the ideal gas law have around 5-10 percent resolution. This is essentially a billion dollar problem that this research group alongside NASA is trying to solve. There are many groups out there working on this problem, however this specific solution is continually supported by NASA due to large success over the years. The method for this solution can get close to 1 percent resolution which is incredible compared to current solutions.

1.3. Concept The concept behind this technology is largely based on resonance and the harmonic oscillator. Liquid in microgravity does not sit at a flat level so conventional methods that are used on Earth are not effective. Zero gravity is a surface tension driven regime, in short, that means the liquid will adhere and rise up the inner walls of the fuel tanks in a microgravity environment. Based on this fact, the technology can take advantage of a variable thickness of the tank walls.

Every object has some sort of natural response frequency. A simple wine glass with liquid inside will project a specific frequency noise if the brim is vibrated correctly. It is not the wine or the glass that responds, it is the air inside the 
glass. The fuel tanks take advantage of this kind of vibrational response but in a different way. These fuel tanks have several modes of vibration. The ones that are important for this technology are the relatively low frequency responses, around 600 to $1000 \mathrm{~Hz}$. These are the radial responses of the tanks. These are the literal expansion and contraction of the thanks, they aren't visible to the human eye, but they are present. The reason that these are observed over the higher frequency responses has to do with electrical noise. These responses are relatively cleaner and comparably much easier to pick out. Now, key reasoning behind these responses has to do with the changing thickness of the tank walls. As more fluid enters the tank, the walls get thicker. The modal responses behave similar to a harmonic oscillator. The thicker the walls, the lower the frequency response of vibration.

$$
\sqrt{k / m}
$$

This reasoning has so far worked very successfully at lower fill levels. That is because as more and more liquid is added to an empty tank, the walls will get thicker. However at higher levels the inner walls are essentially coated, so adding the same amount of liquid is marginal in the relative thickness of the walls. Now, how are these responses measured?

An amplified white noise signal is input via a Lead Zirconate Titanate (PZT) patch. This patch obeys the piezoelectric effect. In simple terms, it is a crystalline structure that if under stress will generate an electric signal and if given an electric signal it will vibrate. There are also multiple sensors on each tank of the same type of material and an additional monitor. The monitor acts as the input signal while the two sensors placed at different locations on the tanks act as the output signal. The white noise induces a stress on the tanks with a large range of input frequencies. Each tank will then vibrate in accordance with its natural modal responses which depend on the effective mass loading, or in short tank wall thickness. To pull out these desired frequencies, the input and the output signals are used in what is called a transfer function. Essentially, this transfer function ratios these two signals after transferring the raw voltage data into the frequency domain. To get to the frequency domain requires a Fourier transform. By taking the ratio of the responses in the frequency domain, the white noise signal is essentially canceled because all that excess noise will appear in both sensors and the monitor. What is left are the additional responses received by the sensors. These are significantly placed further away from the monitor to allow the tank to respond and alter the signal. Each modal response will not be present in the monitor due to a high proximity placement next to the actuator. The resulting response with some noise filtering is shown below in Figure 2.

\section{Parabolic Flights}

The current Carthage College Microgravity Team has been fortunate enough to fly on multiple parabolic flights through Zero G Corporation. The team will fly its experiment for 25-30 parabolas per day. November of 2016 consisted of 2 days, March of 2017 had 4 total days and the upcoming November 2017 flight will have at least 2 days. Each parabola gives the team nearly 20 seconds of zero-gravity during which they run their experiment. Following each parabola is a period of $2 \mathrm{~g}$ where the aircraft pulls up to climb the height it just fell.

2.1. Experimental Payload An image of the experimental payload can be seen in Figure 1. The frame of the payload was constructed using 10 series 80/20 aluminum t-slots, and secondary containment is maintained by gasketed polycarbonate panels on all sides that sit inside the recession of the t-slots. A hinged door at the top of the payload allows access into the interior of the payload. The payload contains two carbon over-wrapped pressure vessels (COPV) connected by a fluid flow loop that allows for automated filling and draining of and fluid transfer between each vessel/propellant tank. Visual confirmation of the fluid levels in each tank is maintained through the use of two liquid level gauges whose bottoms are flush with the bottom of each tank. Both tanks have a set a four piezoelectric patch sensors adhered to their outer walls. The top two sensors are used for data collection, the bottom sensor is the actuator which inputs white noise to the system, and the second patch to the bottom is a monitor, which is used to remove actuator noise from the sensor data.

Data acquisition, signal control, and flow loop control are all maintained by a National Instruments 9133 data acquisition (DAQ) system. The data acquisition system sits in the interior of the payload between the two propellant tanks. An external interface with the team is made possible through a touch screen monitor mounted to the top of the payload. All controls are run from the monitor through LabView. Accompanying the monitor on the top of the payload is an 


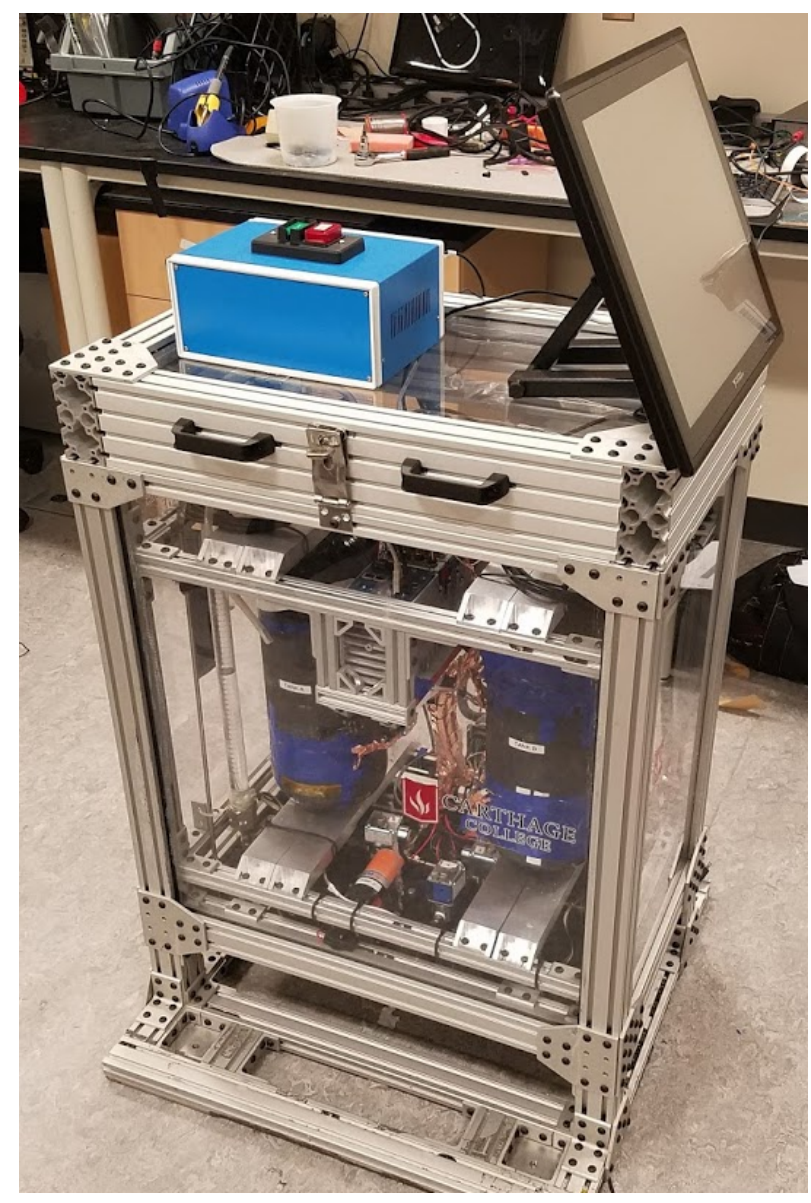

Figure 1: Parabolic flight payload

electronics box which contains all necessary components to regulate power to each electrical system.

2.2. Rig Improvements The payload used in March of 2017 is much different than the payload used in November of 2016. In order to advance this technology on the Technology Readiness Level (TRL) Scale, the experimental rig underwent some changes. The previous rig had 21 -gallon aluminum tanks that were for testing purposes. These were replaced with 2-gallon Composite Over-wrapped Pressure Vessel (COPV) tanks. The COPV tanks are legitimate spaceflight hardware tanks that the team received from a connection at NASA-Kennedy. With COPV tanks, the "experiment" is moving toward a realistic technology. In order to accommodate for much larger tanks in the same frame, a completely redesigned flow loop and inner layout was needed. Over the course of a few months, the new rig was designed and built on the needs presented by these larger tanks. From January to early March, the prior experiment was stripped out and replaced with a brand new system.

Another drastic change was the switch to an internal Data Acquisition System (DAQ). The only access to the inside of the experiment is through a locked lid. This system, the National Instruments cDAQ-9133 runs the experiment software during flights and controls all systems. The parabolic flights are manned-flights so in order to run the software, the DAQ is connected to a touch-screen that is mounted on the exterior of the rig. The previous experiment had the DAQ mounted on the top of the experiment along with a laptop to run the software. This is huge for this project because it shows that the analysis can be done by a chip and this technology can be automated.

The DAQ, the touchscreen, the pumps, the actuators, the lights, and the solenoid valves are all powered through a sophisticated power distribution system that is mounted on the top of the experiment. A few problems were encountered toward the end of the March 2017 flight campaign. The screen and the actuators would periodically cut-out. It was 
determined that both were not receiving enough current. Instead of sharing a parallel circuit with another item that needed the same voltage, the original 115-120VAC source was routed into 4 different systems instead of 2 . This has seemed to solve the large issue of losing power which had corrupted a couple datasets from March. The new system ran all summer without losing power so the upcoming flights should run safely without interruption.

2.3. Data Collection Process The data collection process during the parabolic flights makes a very complicated process quite simple. Because it is a manned rig, those who fly with the experiment must activate the software at the beginning of each parabola. The flight crew informs the passengers when the aircraft is "pushing over", which signifies the start of the zero-g period. The crew then activates the software by pushing a button on the touchscreen to record data for the next 20 seconds of microgravity. The zero-g periods are not perfect, there are often negative impulses which cause the liquid in the tanks to slosh around. To accommodate for this problem, the data for one set fill level is averaged over 5 parabolas. After 5 parabolas, the fill level is changed either during a 2-g pull up or a 1-g rest period. Testing is then resumed for the next set of parabolas. This extra sloshing does not allow the liquid inside the tanks to fully settle and coat the inner walls as desired. Based on this problem, it has been determined that something must be implemented to get more realistic results. It is important to note that the fill levels are approximated by the liquid level gauges that are a part of the flow loop of the experimental rig.

2.4. Analysis The following image is a sample of some data collected from a single tank in 1-g over a range of nearly 50 percent of the tank volume. These are two gallon tanks so 1 percent of each tank is near $80 \mathrm{~mL}$, only a couple of fingers of height on an average graduated cylinder. There are distinct peaks between marked fill levels in the middle of the figure. The technology is able to pick out a difference between a 1 percent change of total volume based on the modal response of the tank. This is a very clean data set from a ground test of the technology. While aboard the aircraft there is a lot more noise from the plane as well from fluid sloshing which does not allow a better resolution. The data from March is still being examined and based on initial results it appears that the sloshing has seriously degraded the resolution of the technology. The entire purpose is to be able to correlate this 1-g response to a 0 -g response in a large effort to develop a usable technology for spacecraft to accurately gauge fuel at low fill levels.

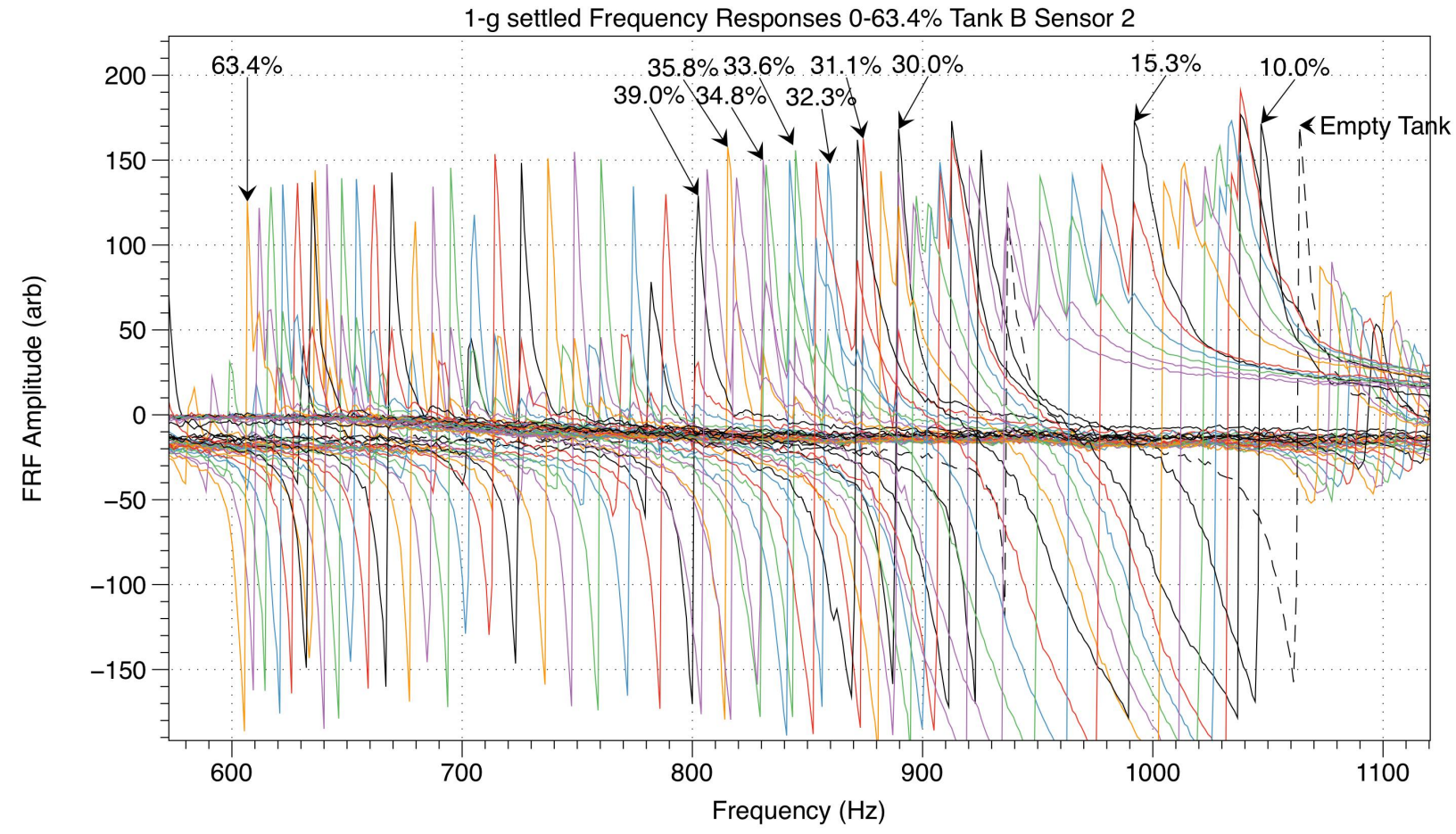

Figure 2: Frequency responses of a $1 \mathrm{~g}$ equilibrium surface 


\section{Anti-Slosh Baffles and CFD}

Our results from previous flights indicate that the fluid in our COPV tanks is not settled by the end of each parabola. The periods of zero gravity last 20-30 seconds. We want to be able to take data while the liquid inside the tanks is settled, simulating the most realistic circumstance as possible. Our idea to solve this problem was to add an anti-slosh baffle system into one of the two COPV tanks in our rig before our next set of parabolic flights.

3.1. Anti-Slosh Baffle Design The current design of the insertable baffle system resembles an upside down umbrella. The baffle system needs to be able to be inserted into one of the COPV tanks without damaging it or disassembling it. The design is also useful since it can be removed somewhat easily from the other end of the tank. The top and bottom holes of the tank are 0.7 inches in diameter and the inner diameter of the tank is $6.7 \mathrm{in}$. The baffle system needs to coat the inner perimeter of the tank in annular rings. It was decided that three baffle rings would be sufficient in preventing slosh.

The anti-slosh baffle system will use nylon fabric rings with an outer diameter equal to the tanks inner diameter. It will be attached by epoxy to spokes made of polypropylene that is perforated, so the liquid is still free to flow, and flexible, so it can fit through the top of the tank. Once it is inserted, the plastic is stiff enough to reopen and the fabric will touch the perimeter of the tank, preventing sloshing. The three sets of rings and spokes will be attached to a in aluminum rod by shafts collars above and below each of them. The anti-slosh baffle system will be built before our next parabolic flights in November.

3.2. Computational Fluid Dynamics Computational fluid dynamic simulations were done to test the design of the anti-slosh baffle system. The simulations were done with SimFlow, which using OpenFoam. The shape of the COPV tanks and the baffle stoke and ring design were recreated on SolidWorks. Simulations were done with and without the baffles in the tanks from fill levels of the tank ranging from ten percent to eighty percent. It was found that the baffles did prevent the liquid from traveling up the sides of the tanks.

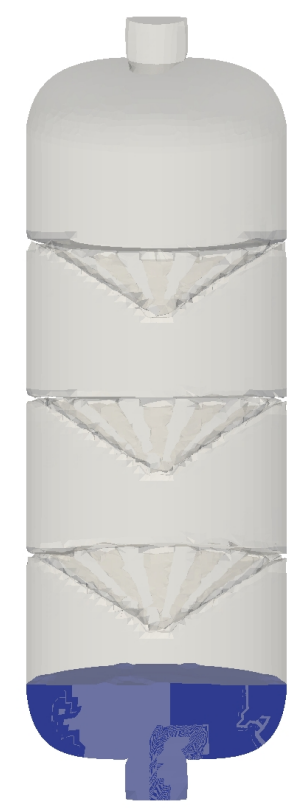

Figure 3: CFD simulations with anti-slosh baffles.

\section{New Shepard Payload}

4.1. New Shepard Introduction The success of the Modal Propellant Gauging project hinges on the hypothesis that the resonant modes seen at varying fluid levels are directly correlated to the effective mass of the propellant tank. 
Because fluid behaves differently in microgravity environments than on Earth, the effective mass of the propellant tank is made up of not only the tank walls, but the layer of fluid that coats the interior of the walls when in microgravity. The fluid coating effectively thickens the tank wall, which is why the combination of the tank walls and the fluid is considered to be the tank's effective mass. The purpose of the New Shepard payload is to test this hypothesis aboard a Blue Origin New Shepard research flight.

Blue Origin's New Shepard is a re-usable, sub-orbital launch vehicle designed to transport a six-man crew to space to experience the wonders of microgravity and the region beyond Earth's lower atmosphere. During manned flights, the crew will experience the journey inside a crew capsule located atop the vehicle. The research flight our payload will fly aboard is an unmanned flight, in which case, the crew capsule will contain payload lockers in place of seats to hold several experiments. Unlike the Zero-G Corporation's parabolic flight campaigns, Blue Origin's New Shepard flight will provide approximately two and a half minutes of uninterrupted microgravity time. This extended duration of microgravity time is essential to the purpose of the payload, as the fluid needs to be in a settled state to test the mass loading hypothesis.

4.2. Payload Concept The team took an "inside-out" design approach for the New Shepard payload, where the payload was designed from the propellant tanks outward. Fluid properties do not scale linearly, so given the dimensional constraints of the payload locker, careful consideration was given when determining the propellant tank size. If the tanks were made to be too small, the properties of the fluid, as well as the fluid dynamics, would deviate from an accurate re-creation of in-flight conditions. If the tanks were made to be too large, the number of propellant tanks contained within the payload would decrease, which in turn would reduce the amount of data we could record. Furthermore, it was important to consider not only the absolute dimensions of the tanks, but the ratio of the height of the tanks to their diameter (cylindrical tanks were used). After running computational fluid dynamics (CFD) simulations, the dimensions of the tanks were chosen to be 10.8" in height and 6" in diameter. Given the size of the propellant tanks, isopropyl alcohol will be used as the model propellant to preserve the scaling properties of the fluid dynamics. The remaining elements of the payload were designed around the size and arrangement of the tanks, and the overall structure was designed to use all of the available interior volume provided by the payload locker.

Unlike the parabolic flight payload, the New Shepard payload will use only one sensor and a monitor, rather than two sensors and a monitor. Additionally, there is no flow loop in the New Shepard payload, so each tank will contain a different, yet fixed fill level. Lastly, the experiment will run autonomously, collecting data throughout the entire duration of the flight. Overall, the payload was designed to be as simple as possible in mechanical and electrical design, as well as concept. Because this will be the first New Shepard flight for the Modal Propellant Gauging team, it was decided that a simpler payload would increase the chance of mission success.

4.3. Payload Design and Construction The payload was made a rectangular cube of nominal dimensions 18 " $\times 14$ " $\times 18$ ". The structural frame of the payload was constructed using 10-series $80 / 20$ aluminum t-slots, and the t-slots are held together by 80/20 aluminum joining plates that use bolts and t-nuts. A labeled diagram of the payload can be found in Figure 4 


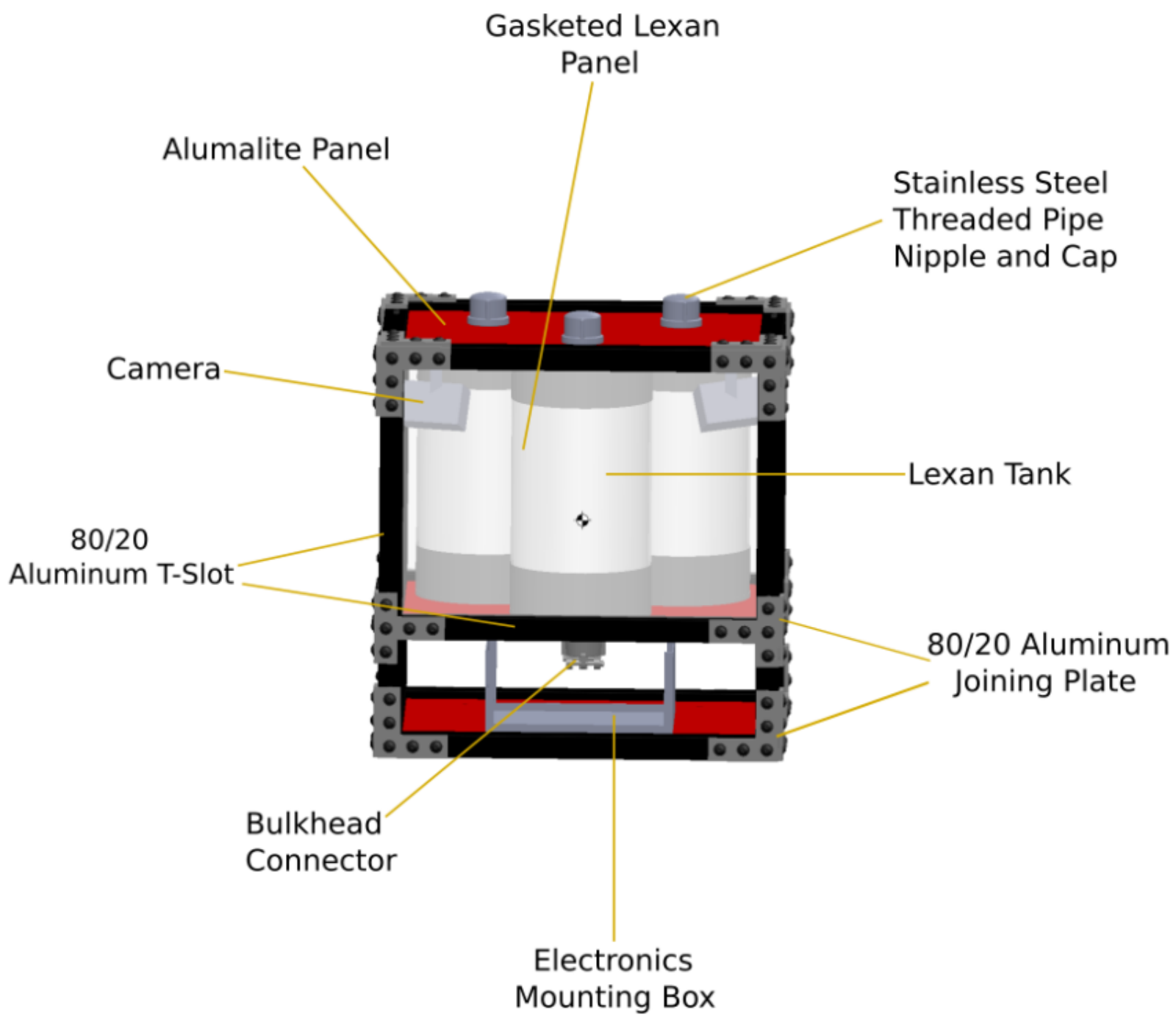

Figure 4: Labeled Solidworks model of New Shepard payload

The payload is divided into two sections: the secondary containment area (top), and the electronics and mounting deck (bottom).

4.3. Secondary Containment Area The Secondary containment area is kept sealed via four gasketed polycarbonate panels on each side, two gasketed Alumalite panels on the top and bottom, and three threaded stainless steel pipe nipples with sealant applied around the base of the nipples where they protrude from the secondary containment area. The pipe nipples allow external access to the interiors of the tanks, but not to the interior of the secondary containment area. Contained within the secondary containment area are three polycarbonate cylindrical propellant tanks arranged in an isosceles triangular formation, cylindrical propellant tank mounting caps, and two GoPro action cameras mounted in the front top corners of the containment area and angled downward and inward at approximately 45-degree angles to allow for a full view of all three propellant tanks. Visual confirmation of the settled state of the propellant is one of the success criteria of the mission, so the secondary containment area will also contain LED lighting strips for interior lighting.

4.3. Electronics and Mounting Deck The data acquisition system, power regulators, white noise generators, and signal amplifiers will all sit inside an aluminum project box mounted to the payload deck via a din rail. All 
required electrical connections will be wired into the secondary containment area via a hermetically sealed bulkhead connector that penetrates the secondary containment area from the bottom at the back of the payload. The payload will be mounted to the locker by bolts directly through the electronics and mounting deck into the threaded holes of the locker's grid pattern. Four bolts will be used in total: one in each corner.

4.3. Construction The payload was initially drafted in Solidworks for verification of dimensions and proof of concept. After the design of the payload was finalized, construction began. Leading up to the research flight in the first half of 2018, construction of the payload will be completed along with full systems integration, full mission simulations, and integration of the payload into the payload locker system. An image of the payload can be found in Figure 5.

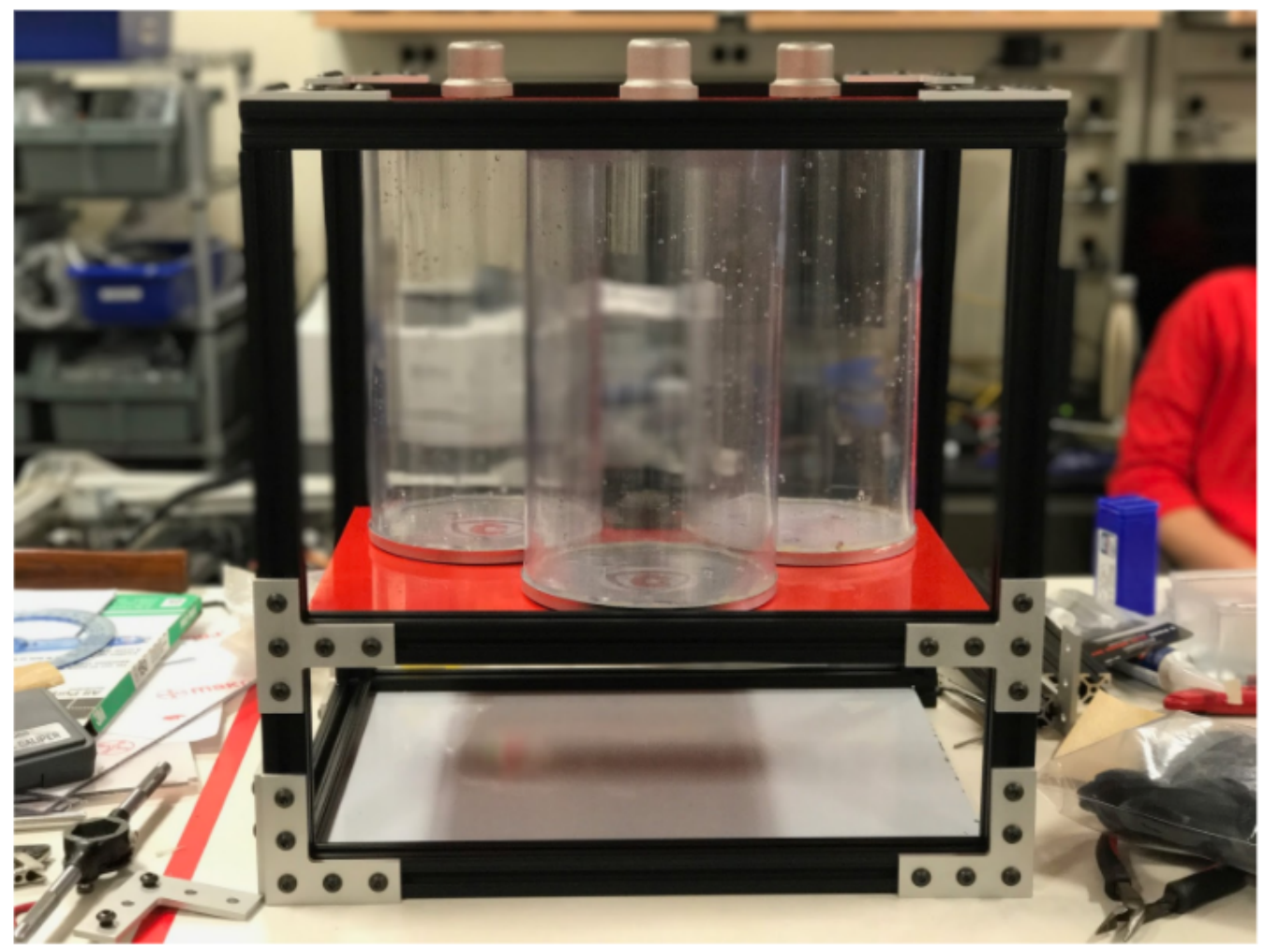

Figure 5: New Shepard payload

\section{Summary}

The MPG project has been around for quite some time. There are a couple of key differences to note about the recent summer months. In order to advance the technology down the TRL pipeline, changes must be made during each iteration to make this into something that hopefully be used on the SLS Orion Missions. The parabolic flight payload has been drastically changed in the last year, there is a completely enclosed operation system that further pushes this to be a technology that can be fully automated. COPV tanks were also used to simulate and collect data on actual spaceflight hardware. It turned out to be very successful, however internal liquid sloshing became apparent to be problematic. To solve this problem, slosh baffles are currently being developed with the help of extensive CFD simulations. The other major project that was worked on this summer was for the Blue Origin New Shepard Mission in 2018. This project is to test the original hypothesis and visually observe the behavior of liquid inside a tank during zero-g. The project is quickly progressing and has been worked on vigorously by students for a number of years. These types of projects are essential to undergraduate learning and provide an amazing experience for many students. 


\section{Acknowledgments}

We would would to thank our advisor and the director of WSGC, Kevin Crosby, for giving us the opportunity to work on this project. Our gratitude is given to Wisconsin Space Grant Consortium for funding this project. We would also like to thank our collaborators at NASA for supplying our COPV tanks to us. Finally, thank you Zero-G corporation for giving us the ability to test our project in a microgravity environment. 